\title{
Local Analytic Solutions of the Generalized Dhombres Functional Equation I
}

By

\section{Ludwig Reich, Jaroslav Smítal, and Marta Stefánková}

(Vorgelegt in der Sitzung der math.-nat. Klasse am 17. März 2005

durch das w. M. Ludwig Reich)

\begin{abstract}
We study local analytic solutions $f$ of the generalized Dhombres equation $f(x f(x))=$ $\varphi(f(x))$ with $f(0)=0$ in the complex domain. We give an existence result, describe the structure of the set of all local analytic solutions and solve the converse problem, i.e., we characterize those local analytic functions which are solutions of a generalized Dhombres equation. Connections of generalized Dhombres equations with linear functional equations and generalized Böttcher equations are used. Furthermore, we establish relations of generalized Dhombres equations with Briot-Bouquet differential equations and with iteration groups. Finally, as an application of Böttcher functions, we describe the connections between two generalized Dhombres equations and the representations of their solutions as infinite products.
\end{abstract}

Mathematics Subject Classification (2000): Primary 30D05, 34M25, 39B12, 39B32; Secondary 30B 10 .

Key words and phrases: Iterative functional equation, locally analytic solutions.

\section{Introduction}

Continuous solutions of a generalization of the Dhombres functional equation (cf. [1]) on real intervals and their dynamics have been studied in several papers (e.g., [4], [5], [12]), in particular recently in [13]. 
However, also the problem of the existence of analytic (holomorphic) solutions of such functional equations seems to be interesting and promising, as well as the study of the dynamics of these equations in the complex domain. As we will see in this note, the generalized Dhombres functional equations are closely related to certain generalized Böttcher equations which were recently investigated in [11] by methods from complex analysis.

We are going to investigate functional equations of the type

$$
f(x f(x))=\varphi(f(x)),
$$

where $\varphi$ is a given function and $f$ denotes the solution. In the present situation we will assume that

$$
\begin{gathered}
\varphi \text { is holomorphic at } x=0, \\
f \text { is holomorphic at } x=0 \text { and } f(0)=0,
\end{gathered}
$$

(i.e., 0 is a fixed point of the solution).

We are going to investigate local analytic solutions $f$ of (1). If $\varphi$ is holomorphic for $|x|<\delta$ (with some $\delta>0$ ), then by a local analytic solution of (1) we understand a function $f$ with $f(0)=0$, analytic in $|x|<\varepsilon$ (for some $\varepsilon>0$ ), such that (1) holds for $|x|<\varepsilon$. This $\varepsilon$ may and will depend on the particular solution $f$. We will not approach here the problem to find an explicit estimate for $\varepsilon=\varepsilon(f)$.

We give now some necessary conditions on $\varphi$ and $f$ which have to be fulfilled if (1) has a local analytic solution $f$ such that (2) and (3) hold, and such that $f \neq 0$ (i.e., $f$ is "nontrivial").

Remark 1. a) Let $f$ be a local analytic solution of (1), i.e., (2) and (3) are satisfied. Then $\varphi(0)=0$.

b) If $\varphi=0$, then $f=0$ is the only local analytic solution of (1) in each neighbourhood of $x=0$.

c) Let $f \neq 0$ be a local analytic solution of (1) such that (2) and (3) are satisfied. Then there exist an integer $k \geq 1$ and $c_{k} \in \mathbb{C} \backslash\{0\}=: \mathbb{C}$ such that

$$
\varphi(x)=x^{k+1}+d_{k+2} x^{k+2}+\cdots, \quad|x|<\delta,
$$

and

$$
f(x)=c_{k} x^{k}+c_{k+2} x^{k+2}+\cdots, \quad|x|<\varepsilon,
$$

for some $\delta>0$ and $\varepsilon=\varepsilon(f)>0$.

Proof. a) This is obvious. b) Let $f$ be a local analytic solution of (1). Assume that $f \neq 0$. This means $f(0)=0$ and $f(x f(x))=0$ in some 
neighborhood of $x=0$ and there exists an integer $k \geq 1$ and $c_{k} \in \mathbb{C}^{\text {. }}$ such that

$$
f(x)=c_{k} x^{k}+\cdots, \quad|x|<\varepsilon .
$$

Then (1) gives

$$
c_{k}^{k+1} x^{k(k+1)}+\cdots=0, \quad|x|<\varepsilon
$$

with $c_{k}^{k+1} \neq 0$. Because of the identity theorem for analytic functions this is a contradiction and hence, $f=0$.

c) Let $f$ be a local analytic solution of (1) with $f \neq 0$. Then, by b), $\varphi \neq 0$. Hence there exist $l \in \mathbb{N}, d_{l} \in \mathbb{C}^{\prime}, k \in \mathbb{N}, c_{k} \in \mathbb{C}^{\prime}$ such that

$$
\varphi(x)=d_{l} x^{l}+\cdots, \quad|x|<\delta,
$$

and

$$
f(x)=c_{k} x^{k}+\cdots, \quad|x|<\varepsilon,
$$

for some $\delta>0, \varepsilon>0$. Then (1) yields that on the left-hand side $f(x f(x))$ has order $k(k+1)$ at $x=0$, and $f(x f(x))=c_{k}^{k+1} x^{k(k+1)}+\cdots$ while, on the right-hand side, $\varphi(f(x))$ has order $l k$, and $\varphi(f(x))=d_{l} c_{k}^{l} x^{l k}+\cdots$.

Hence $l k=k(k+1)$ which gives $l=k+1$ and $c_{k}^{k+1}=d_{k+1} c_{k}^{k+1}$. Since $c_{k}^{k+1} \neq 0$ we have $d_{k+1}=1$, and $\varphi(x)=x^{k+1}+d_{k+2} x^{k+2}+\cdots$, for $|x|<\delta$. This proves $\mathrm{c}$ ), and hence, the argument is complete.

The paper is organized as follows. In Sect. 2 we show the existence of locally analytic solutions of (1), provided $\varphi(x)=x^{k+1}+\cdots$ is analytic at $x=0$. We show that, for each $c_{k} \in \mathbb{C}$, there exists exactly one local analytic solution $f(x)=c_{k} x^{k}+\cdots$ (Theorem 1). Then we show that every formal solution $f(x)=c_{k} x^{k}+\cdots$ of (1) is convergent by applying results on local analytic solutions of the generalized Böttcher equation. A different representation of local analytic solutions of (1) in our situation is given in Theorem 2, by using the classical iteration process for a linear functional equation to which (1) can be reduced. This representation involves certain infinite products.

In Sect. 3 we give as a consequence of Theorem 1 and its proof a result on the structure of the set of all local analytic solutions of (1), for $\varphi \neq 0$ (Theorem 3).

This structure theorem leads to the answer of a converse problem: For which $f \neq 0$ there exist $\varphi$ such that $f$ is a solution of (1)? The answer is formulated in Theorem 4 . Roughly speaking, the structure derived for the set of local analytic solutions of (1) is also sufficient to have a set of local analytic functions being the solutions of a generalized Dhombres equation. This converse problem, in the real case, was solved in [13]. 
In Sect. 4 we characterize the set of local analytic solutions of (1) as the set of all local analytic solutions of a certain Briot-Bouquet differential equation, associated with (1) in a unique way (Theorem 5; for Briot-Bouquet differential equations see [3], [7], [2]). Conversely, we associate to each Briot-Bouquet differential equation of the form $x w^{\prime}(x)=k \cdot N(w(x))$ a unique generalized Dhombres equation (1) so that the sets of local analytic solutions are the same.

In Sect. 5 we establish a connection between the set of all local analytic solutions of (1) for $\varphi \neq 0$, with analytic iteration groups of first type. This gives a parametrization of the set of local analytic solutions of (1) as members of such an iteration group (Theorem 7). The proof is based on the characterization of iteration groups of type I by certain Aczél-Jabotinsky differential equations which can be equivalently transformed to the class of Briot-Bouquet differential equations occurring in Sect. 4.

Conversely, we associate with each analytic iteration group of type I an equation (1) such that the set of local analytic solutions of this (1) consists exactly of the members of the given iteration group.

Moreover, the group operation of the iteration group associated with (1) induces a group operation on the set of local analytic solutions of (1) which takes the form of a perturbed translation equation.

In Sect. 6 we apply Böttcher functions (i.e., the classical Böttcher equation) to equations (1). This leads to another representation of the non-trivial local solutions of (1) (Theorem 9), but also to the explicit construction of a bijection between the sets of solutions of two generalized Dhombres equations (1), with functions $\varphi_{1}$ and $\varphi_{2}$ on the right-hand side, in the case when

$$
\varphi_{j}(x)=x^{k+1}+\cdots, \quad j=1,2,
$$

with the same $k \geq 1$ (Theorem 10).

\section{Local Analytic Solutions of Generalized Dhombres Equations with Fixed Point 0}

From Remark 1 we know that in a generalized Dhombres equation (1) with non-trivial local analytic solutions $f$ with $f(0)=0$ we necessarily have $\varphi(x)=x^{k+1}+d_{k+2} x^{k+2}+\cdots,|x|<\delta$ for some $k \geq 1$, and that then necessarily $f(x)=c_{k} x^{k}+\cdots$, with $c_{k} \neq 0$ and $|x|<\varepsilon$. The existence problem is solved by

Theorem 1. a) Let $\varphi(x)=x^{k+1}+\cdots$ with $k \geq 1$ be analytic at $x=0$. Then to each $c_{k} \in \mathbb{C}$ there exists exactly one solution $f$ of (1) with $f(x)=c_{k} x^{k}+\cdots,|x|<\varepsilon$. 
b) All local analytic solutions $f$ with $f(0)=0$ of (1) are given by a), $c_{k}=0$ if and only if $f=0$.

Proof. Let $f$ be a local analytic solution of (1) with $f(0)=0$. If $f \neq 0$, then by Remark 1 there exists $k \geq 1$ such that $f(x)=c_{k} x^{k}+\cdots$, with $c_{k} \neq 0$. Hence $f=0$ means $c_{k}=0$. Our proof is based on the following two remarks.

Remark 2. Let $k \geq 1$, and $f$ with $f(x)=c_{k} x^{k}+\cdots, c_{k} \neq 0$, be analytic at $x=0$. Then there exist exactly $k$ different local analytic functions $T^{(l)}(x), l=0, \ldots, k-1$, such that

$$
\left(T^{(l)}(x)\right)^{k}=f(x)
$$

holds in some neighbourhood of $x=0$. We have $T^{(l)}(x)=t_{1}^{(l)} x+\cdots$ with $t_{1}^{(l)} \neq 0$, for $l=0, \ldots, k-1$. These functions $T^{(l)}$ may be arranged so that $T^{(l)}(x)=\zeta^{l} T^{(0)}(x)$, where $\zeta=e^{2 \pi i / k}$. Each $T^{(l)}$ is uniquely determined by its linear part $t_{1}^{(l)}(x)$, and $c_{k}=\left(t_{1}^{(l)}\right)^{k}$, for $l=0, \ldots, k-1$.

This result is kind of folklore (see [14], pp. 223-224) but we include here a proof for the convenience of the reader. Assume that $T(x)$, analytic at $x=0$, is a solution of $T(x)^{k}=f(x)$ in a neighbourhood of 0 . Then $T(x)=t_{1} x+\cdots$ with $t_{1}^{k}=c_{k}$. Write $T(x)=t_{1} x\left(1+\tilde{t}_{1} x+\cdots\right)$ and $f(x)=c_{k} x^{k}\left(1+\tilde{c}_{1} x+\cdots\right)$. Then

$$
t_{1}^{k} x^{k}\left(1+\tilde{t}_{1} x+\cdots\right)^{k}=c_{k} x^{k}\left(1+\tilde{c}_{1} x+\cdots\right)
$$

in a neighbourhood of 0 , hence for $x \neq 0$,

$$
\left(1+\tilde{t}_{1} x+\cdots\right)^{k}=1+\tilde{c}_{1} x+\cdots, \quad 0<|x|<\varepsilon .
$$

This relation can be holomorphically continued to $x=0$, and the unique solution $1+\tilde{t}_{1} x+\cdots$ is obtained by substituting $\tilde{c}_{1} x+\tilde{c}_{2} x^{2}+\cdots$ into the binomial series $(1+y)^{1 / k}$. Since $t_{1}^{k}=c_{k}$ has exactly $k$ different complex solutions $t_{1}$, Remark 2 is proved.

Remark 3. Let $k \geq 1, l \geq 1$, and let $F(x)=x^{l k}+c_{l k+1} x^{l k+1}+\cdots$ be analytic at $x=0$. Then there exists a unique $G(x)=x^{l}+$ $D_{l+1} x^{l+1}+\cdots$, analytic at $x=0$, such that $G(x)^{k}=F(x)$ holds in some neighbourhood of 0 .

Now to prove a) let $f$ be a solution of (1), $f(x)=c_{k} x^{k}+\cdots, c_{k} \neq 0$. Then, according to Remark 2 there exists $T(x)=t_{1} x+\cdots$ with $t_{1} \neq 0$ and analytic at $x=0$, such that $F(x)=T(x)^{k}$ holds in some neighbourhood of 0 . Substituting this into (1) we find, for sufficiently small $|x|$,

$$
\left(T\left(x T(x)^{k}\right)\right)^{k}=\varphi\left(T(x)^{k}\right) .
$$


Since the local (analytic) inverse $T^{-1}$ of $T$ exists, we can substitute $T^{-1}(x)$ for $x$ in the last relation and obtain

$$
\left(T\left(T^{-1}(x) \cdot x^{k}\right)\right)^{k}=\varphi\left(x^{k}\right)
$$

in some neighbourhood of $0 . T\left(T^{-1}(x) x^{k}\right)$ has a Taylor series in $x$ starting with $x^{k+1}$. Hence by Remark $3, T\left(T^{-1}(x) x^{k}\right)=\psi(x)$ in some neighbourhood of 0 , where $\psi$ is uniquely determined by

$$
\psi(x)^{k}=\varphi\left(x^{k}\right), \quad \psi(x)=x^{k+1}+\cdots,
$$

for sufficiently small $|x|$. Applying $T^{-1}$ on both sides of (15) we get, with $\mathcal{U}=T^{-1}$,

$$
x^{k} \mathcal{U}(x)=\mathcal{U}(\psi(x))
$$

in some neighbourhood of 0 , with $\mathcal{U}(x)=u_{1} x+\cdots, u_{1} \neq 0$. Then (17) is locally equivalent with (1), and may be viewed either as a linear functional equation (see [6]) or a generalized Böttcher equation for $\mathcal{U}$ (cf. [11]).

We take now the second point of view. From Theorem 7 of [11] it follows that for each $u_{1} \in \mathbb{C}^{\cdot}$ there exists exactly one local analytic solution $\mathcal{U}(x)=u_{1} x+\cdots$ of (17), and these are all such solutions with $\mathcal{U}(0)=0, \mathcal{U} \neq 0$. It is now clear that with $T=\mathcal{U}^{-1}, f(x)=(T(x))^{k}$ yields a nontrivial solution of $(1)$, and $f(x)=\left(1 / u_{1}^{k}\right) x^{k}+\cdots$. By an appropriate choice of $u_{1}$ we get each value $c_{k}=1 / u_{1}^{k}$ in $\mathbb{C}$.

It remains to prove that a solution $f$ of (1) with $f(x)=c_{k} x^{k}+\cdots$ is uniquely determined by $c_{k}$. Assume that $f_{1}$ and $f_{2}$ are both solutions of (1) with

$$
f_{j}(x)=c_{k} x^{k}+\cdots, \quad(j=1,2)
$$

with the same $c_{k}$. Take $T_{1}$ and $T_{2}$ according to Remark 2, so that

$$
\left(T_{j}(x)\right)^{k}=f_{j}(x), \quad j=1,2
$$

in some neighbourhood of 0 , and such that $T_{1}$ and $T_{2}$ have the same linear part $t_{1} x$. Then $\mathcal{U}_{1}=T_{1}^{-1}$ and $\mathcal{U}_{2}=T_{2}^{-1}$ are solutions of (17) with the same linear part, and hence $\mathcal{U}_{1}=\mathcal{U}_{2}, T_{1}=T_{2}$ and $f_{1}=f_{2}$. This proves Theorem 1a).

Theorem $1 \mathrm{~b}$ ) is clear, since the representation $f=T^{k}$ is possible for all local analytic solutions $f$ of (1) with $f \neq 0, f(0)=0$. This completes the proof of Theorem 1 .

The essential step for the proof of Theorem 1 was to solve (17). We now consider (17) as a linear functional equation. In order to construct all local analytic solutions $\mathcal{U} \neq 0$ of (17) we make some more 
preparatory transformations. We put $\mathcal{U}(x)=u_{1} x \cdot \tilde{\mathcal{U}}(x)$ in a neighbourhood of $x=0$, so that $\tilde{\mathcal{U}}(x)=1+\cdots$. Then (17) is equivalent with

$$
\tilde{\mathcal{U}}(\psi(x))=\frac{x^{k+1}}{\psi(x)} \cdot \tilde{\mathcal{U}}(x)
$$

in some neighbourhood of 0 , where $\theta: x \mapsto x^{k+1} / \psi(x)$ is, by holomorphic extension to $x=0$, analytic at $x=0$, and $x^{k+1} / \psi(x)=1+\cdots$.

Now the classical "iteration process" (cf. [6], Ch. 3) may be applied to the linear equation (20) and yields

$$
\tilde{\mathcal{U}}(x)=\prod_{\nu=0}^{\infty} \frac{\psi^{\nu+1}(x)}{\left(\psi^{\nu}(x)\right)^{k+1}},
$$

which converges uniformly and absolutely in each compact subset of a certain neighbourhood of 0 . Here $\psi^{\nu}$ is the $\nu$-th iterate of $\psi$, and these iterates are analytic in a common neighbourhood of 0 , since $\psi(x)=x^{k+1}+\cdots$, with $k+1 \geq 2$. Putting everything together we get

Theorem 2. Let $\varphi(x)=x^{k+1}+\cdots$ be analytic at $x=0, k \geq 1$. Then the set of local analytic solutions $f$ of (1), with $f \neq 0$ and $f(0)=0$, is given by

$$
f(x)=\left(\left[u, x \cdot \prod_{\nu=0}^{\infty} \frac{\psi^{\nu+1}(x)}{\left(\psi^{\nu}(x)\right)^{k+1}}\right]^{[-1]}\right)^{k}
$$

in some neighbourhood $|x|<\varepsilon(f)$. Here $\psi^{\nu}$ is the $\nu$-th iterate of $\psi$. $[\theta(z)]^{[-1]}$ denotes the inverse of $\theta$.

\section{The Structure Theorem. A Converse Problem}

Let $\varphi(x)=x^{k+1}+d_{k+2} x^{k+2}+\cdots$ be analytic in $x=0$, with $k \geq 1$. Now we will prove that the set of all local solutions $f(x)=$ $c_{k} x^{k}+\cdots\left(c_{k} \in \mathbb{C}\right)$ has the following structure.

Theorem 3. Let $\varphi(x)=x^{k+1}+d_{k+2} x^{k+2}+\cdots$ be analytic in $x=0$, with $\underset{\tilde{f}}{k} \geq 1$. Then there exists exactly one local analytic function $\tilde{f}_{0}$, with $\tilde{f}_{0}(x)=x+\cdots$, such that the set of all local analytic solutions $f(x)=c_{k} x^{k}+\cdots$ of (1) has the form

$$
\left\{f \mid f(x)=\tilde{f}_{0}\left(c_{k} x^{k}\right), c_{k} \in \mathbb{C}\right\}
$$

for $|x|<\varepsilon(f) ; \varepsilon(f)$ depending in general on $f$.

Proof. We know from Theorem 1 that under our hypothesis on $\varphi$ to each $c_{k} \in \mathbb{C}$ there exists exactly one local analytic solution $f$ of (1) 
with $f(x)=c_{k} x^{k}+\cdots(|x|<\varepsilon(f))$, and that these are all solutions of (1). In order to obtain the solutions $f \neq 0$ we wrote $f(x)=(T(x))^{k}$ and got for $T^{-1}=\mathcal{U}$ the functional equation (17) in some neighbourhood of 0 , where $\psi(x)$ was uniquely determined from $\psi(x)^{k}=\varphi(x)^{k}$, $\psi(x)=x^{k+1}+\cdots$. We claim that $\psi$ has the form

$$
\psi(x)=z \cdot \tilde{\psi}\left(x^{k}\right)
$$

in a neighbourhood of 0 where $\tilde{\psi}(x)=x+\cdots$. To see this write $\varphi(x)=x^{k+1} \tilde{\varphi}(x)$ with $\tilde{\varphi}(x)=1+\cdots, \quad$ and hence $\varphi\left(x^{k}\right)=$ $x^{k(k+1)} \tilde{\varphi}\left(x^{k}\right)$. Hence (using Remark 3) $\psi(x)=x^{k+1} \tilde{\varphi}\left(x^{k}\right)^{1 / k}$ where we now use the binomial series for the exponent $1 / k$ to get $\psi(x)=$ $x \cdot x^{k} \cdot \tilde{\psi}\left(x^{k}\right)=x \cdot \tilde{\psi}\left(x^{k}\right)$ with $\tilde{\psi}(x)=x+\cdots$ Using the form (24) of $\psi$ and substituting $\zeta x$ with $\zeta=e^{2 \pi i / k}$ in (1) we find

$$
x^{k} \mathcal{U}(\zeta x)=\mathcal{U}\left(\zeta x \tilde{\psi} \cdot\left(x^{k}\right)\right) .
$$

This means that $x \longmapsto \mathcal{U}(\zeta x)$ is a local analytic solution of (17) such that $\mathcal{U}(\zeta x)=\zeta u_{1} x+\cdots$, if $\mathcal{U}(x)=u_{1} x+\cdots, u_{1} \neq 0$. Since (1) is a linear functional equation for $\mathcal{U}$ and since a solution of (1) is uniquely determined by its first coefficient we get $\mathcal{U}(\zeta x)=\zeta \mathcal{U}(x)$ in some neighbourhood of 0 , hence

$$
\mathcal{U}(x)=\sum_{\nu=0}^{\infty} u_{\nu k+1} x^{\nu k+1} .
$$

Now we show that $T=\mathcal{U}^{-1}$ has the same structure. $T$ is characterized by the relation $T(\mathcal{U}(x))=x$ near 0 . Again substituting $\zeta x$ for $x$ by (26) we get $T(\mathcal{U}(\zeta x))=T(\zeta \mathcal{U}(x))=\zeta x$. Again by substituting $T(x)$ for $x$ we find, because of $\mathcal{U}(T(x))=x$, that $T(\zeta x)=\zeta T(x)$ in some neighbourhood of 0 , hence

$$
T(x)=\sum_{\nu=0}^{\infty} t_{\nu k+1} x^{\nu k+1}=t_{1} x \cdot \tilde{T}\left(x^{k}\right),
$$

where $t_{1}=1 / u_{1}$ and $\tilde{T}(y)=1+\cdots$.

Now let $\mathcal{U}^{(0)}$ be the unique solution of (1) with $\mathcal{U}^{(0)}(x)=x+\cdots$, and $T^{(0)}=\left(\mathcal{U}^{(0)}\right)^{-1}$. Since (1) is linear and each solution $\mathcal{U}(x)=$ $u_{1} x+\cdots$ of (1) is determined by its first coefficient $u_{1}$, we get $\mathcal{U}=u_{1} \mathcal{U}^{(0)}$, and for $T=\mathcal{U}^{-1}$

$$
T(x)=T^{(0)}\left(t_{1} x\right)
$$

with $t_{1}=1 / u_{1}$. Indeed,

$$
T^{(0)}\left(t_{1} \mathcal{U}(x)\right)=T^{(0)}\left(\frac{1}{u_{1}} \mathcal{U}(x)\right)=T^{(0)}\left(\mathcal{U}^{(0)}(x)\right)=x,
$$


in some neighbourhood of 0 , so that the local inverse $T$ of $\mathcal{U}$ is given by (28). Now, since $f(x)=(T(x))^{k}$ we find by (27) and (28),

$$
f(x)=(T(x))^{k}=\left(T^{(0)}\left(t_{1} x\right)\right)^{k}=\left(t_{1} x \cdot \tilde{T}^{(0)}\left(t_{1} x\right)^{k}\right)=\tilde{f}_{0}\left(c_{k} x^{k}\right),
$$

where, using $(27), T^{(0)}(x)=x \cdot \tilde{T}^{(0)}\left(x^{k}\right), c_{k}=t_{1}^{k}$, and $\tilde{f}_{0}(y):=y \cdot \tilde{T}^{0}(y)$, which only depends on $\varphi$, but not on the individual solution $f$. Moreover, $\tilde{f}_{0}(y)=y+\cdots$. It remains to prove that $\tilde{f}_{0}$ is uniquely determined by $\varphi$, i.e., by the given functional equation (1). In fact, it is uniquely determined by any non-trivial solution $f(x)=c_{k} x^{k}+\cdots$, $k \geq 1, c_{k} \neq 0$, of (1). Assume, that in some neighbourhood of 0 , we have

$$
f(x)=\tilde{f}_{0}\left(c_{k} x^{k}\right), \quad f(x)=\tilde{g}_{0}\left(c_{k} x^{k}\right), \quad|z|<\eta,
$$

with analytic functions $\tilde{f}_{0}, \tilde{g}_{0}$. Then $\left(\tilde{f}_{0}-\tilde{g}_{0}\right)\left(c_{k} x^{k}\right)=0,|x|<\eta$. Since $c_{k} \neq 0$, the values $c_{k} x^{k}$ cover a neighbourhood of 0 , if $x$ runs through a neighbourhood of 0 . So, by the identity theorem, we get $\tilde{f}_{0}=\tilde{g}_{0}$. Now Theorem 3 is completely proved.

There is the following converse problem (in the real case, cf. [13]): For which functions $f \neq 0$ does there exist a function $\varphi$ such that $f(x f(x))=\varphi(f(x))$ holds in some domain? In our present situation $f$ is a local analytic function, $f(x)=c_{k} x^{k}+\cdots, k \geq 1, c_{k} \neq 0$, in some neighbourhood of 0 . Theorem 3 allows us to answer this question completely for local analytic functions. We have

Theorem 4. a) Let $\tilde{f}_{0}, \tilde{f}_{0}(y)=y+\cdots$, be analytic in some neighbourhood of 0 . Then there exists a unique local analytic $\varphi, \varphi(x)=$ $x^{k+1}+\cdots$, such that the set of all local analytic solutions of (1) is given by

$$
\left\{f\left|f(x)=\tilde{f}_{0}\left(c_{k} x^{k}\right),\right| x \mid<\varepsilon(f), c_{k} \in \mathbb{C}\right\} .
$$

b) Let $f(x)=c_{k} x^{k}+\cdots$ with $k \geq 1, c_{k} \neq 0$ be analytic in some neighbourhood of 0 . Then there exists a local analytic $\varphi$ with $\varphi(0)=0$ such that $f$ is a solution of (1) if and only if $f$ has the form $f(x)=\tilde{f}\left(x^{k}\right)$ in some neighbourhood of 0 , where $\tilde{f}$ is analytic. If such $a \varphi$ exists, then it is uniquely determined by $f$.

Proof. a) Assume that $\varphi$ is a local analytic function with $\varphi(0)=0$, such that each $f$ with $f(x)=\tilde{f}_{0}\left(c_{k} x^{k}\right),|x|<\varepsilon(f)$, is a solution of (1). Then $f(x f(x))=\varphi(f(x)) \Leftrightarrow \tilde{f}_{0}\left(x^{k} \tilde{f}_{0}\left(x^{k}\right)^{k}\right)=\varphi\left(\tilde{f}_{0}\left(x^{k}\right)\right)$ in some neighbourhood of $x=0$. Since $x^{k}$ covers a neighbourhood of 0 , if $x$ runs through a neighbourhood of 0 , we find from this equivalence: $f(x f(x))=\varphi(f(x))$ in some neighbourhood of $x=0 \Leftrightarrow \tilde{f}_{0}\left(y \tilde{f}_{0}(y)^{k}\right)=$ $\varphi\left(\tilde{f}_{0}(y)\right)$ in some neighbourhood of $y=0$. 
Putting $\left(\tilde{f}_{0}^{-1}\right)(y)$ in place of $y$ in the last relation, we get: $f(x f(x))=\varphi(f(x))$ in a neighbourhood of $0 \Leftrightarrow$

$$
\tilde{f}_{0}\left(\tilde{f}_{0}^{-1}(y) \cdot y^{k}\right)=\varphi(y) \text {, in some neighborhood of } 0 .
$$

This shows that $\varphi$ is uniquely determined by $\tilde{f}_{0}$, if it exists. On the other hand, if we define $\varphi$ by (32), then it obviously satisfies the assertions of Theorem 4 a). So, for $\varphi$ defined according to (32), we see that all functions $x \longmapsto \tilde{f}_{0}\left(c_{k} x^{k}\right),|x|<\varepsilon(f)$, are solutions of (1). These are, in fact, all local analytic solutions $f$ of (1) with $f(0)=0$. Indeed, by Theorem 1, there is exactly one solution $f(x)=c_{k} x^{k}+\cdots$ with given $c_{k} \in \mathbb{C}$. But since $\tilde{f}_{0}\left(c_{k} x^{k}\right)=c_{k} x^{k}+\cdots$ and is a solution of (1), we got all solutions.

b) We only have to show the existence of $\varphi$ to a given $f$ and its uniqueness. But this is done with the same calculations as used in the proof of a). This completes the proof of Theorem 4 .

Remark 4. Theorems 3 and 4 show that for the local analytic solutions $f$ with $f(0)=0$ of (1) there may not exist a common region containing 0 where they all are analytic. Take, in Theorem $4 \mathrm{a}), \tilde{f}_{0}$ with $\tilde{f}_{0}(y)=y+\cdots$, so that its radius of convergence is finite (but clearly positive). Then the radius of convergence of $\tilde{f}_{0}\left(c_{k} x^{k}\right)$ tends to 0 with $\lim \left|c_{k}\right|=\infty$. But all functions $x \longmapsto \tilde{f}_{0}\left(c_{k} x^{k}\right)$ are solutions of the same (1).

Remark 5. One may ask for an explicit representation of the function $\tilde{f}_{0}$ in Theorem 3, associated with a given $\varphi$. We may use a similar approach as given in Theorem 2 for the solutions $f$ of (1). Since $\tilde{f}_{0}\left(x^{k}\right)$ is a solution of (1), namely the one with $\tilde{f}_{0}\left(x^{k}\right)=x^{k}+\cdots$ we get from (1), by known calculations and arguments already used before,

$$
\tilde{f}_{0}\left(y \tilde{f}_{0}(y)^{k}\right)=\varphi\left(\tilde{f}_{0}(y)\right)
$$

in some neighbourhood of $y=0$ (cf. (32)) or, equivalently,

$$
y^{k} \tilde{g}_{0}(y)=\tilde{g}_{0}(\varphi(y))
$$

in some neighbourhood of 0 , for $\tilde{g}_{0}=\tilde{f}_{0}^{-1}$. Then (33) is a further generalization of Dhombres' equation, and (34) may be viewed as a generalized Böttcher equation or as a linear functional equation for $\widetilde{g}_{0}, \tilde{g}_{0}(y)=y+\cdots$. Then (34) is the functional equation (17) with $\varphi$ in place of $\psi$. Hence the usual "iteration process" yields

$$
\tilde{g}_{0}(y)=\left[y \prod_{r=0}^{\infty} \frac{\varphi^{r+1}(y)}{\left(\varphi^{r}(y)\right)^{k+1}}\right]^{[-1]} .
$$


Remark 6. In order to describe the local analytic solutions of (1) in our present situation, one could also follow another way:

(i) Show directly from (1), by comparing coefficients of powers $x^{\nu}$ on both sides, that there is a unique formal solution $f(x)=c_{k} x^{k}+\cdots$ with given $c_{k}$ (taking $\left.\varphi(x)=x^{k+1}+\cdots\right)$.

(ii) Then put $f(z)=\tilde{f}\left(z^{k}\right)$ with some $\tilde{f}$ with $\tilde{f}_{1}(y)=c_{k} y+\cdots$. This leads to the functional equation (34) for $\tilde{g}:=\tilde{f}^{-1}$ in place of $\tilde{g}_{0}$ which is equivalent with (1) in some neighbourhood of 0 . Then (34) is the same as (1) with $\varphi$ instead of $\psi$, and we know already that such a functional equation has a unique local analytic solution $\tilde{g}$ with prescribed initial part $\gamma_{1} y$. Hence there exists a unique local analytic solution $f$ of (1) with $f(x)=\tilde{f}\left(x^{k}\right)$ and $\tilde{f}(y)=c_{k} y+\cdots$, where $c_{k} \in \mathbb{C}$ is arbitrarily given. So we get directly to the representation of Theorem 3.

\section{Generalized Dhombres Equations and Briot-Bouquet Differential Equations}

We will now present a formulation of the equations (1) as certain differential equations in complex domain, namely as special Briot-Bouquet differential equations. For this type of differential equations we refer the reader to [3], pp. 295-297, [2], pp. 402-407, [7], pp. 104-106 and [8], pp. 160-162. First, we associate a given (1) with a Briot-Bouquet differential equation.

Theorem 5. a) Let $\varphi(z)=z^{k+1}+d_{k+2} z^{k+2}+\cdots(k \geq 1)$ be analytic in a neighbourhood of 0 . Then there exists a unique local analytic function $N_{\varphi}, N_{\varphi}(z)=z+\cdots$ such that the set of all local analytic solutions of (1) with $f(0)=0$ is the same as the set of local analytic solutions $w$ with $w(0)=0$ of the differential equation

$$
z w^{\prime}(z)=k N_{\varphi}(w(z))
$$

in a neighbourhood of 0 .

b) If we represent, according to Theorem $3 b$ ), the solutions of (1) in the form $f(z)=\tilde{f}\left(z^{k}\right)$, then $\tilde{f}$ is a local analytic solution of the differential equation

$$
z \tilde{w}^{\prime}(z)=N_{\varphi}(\tilde{w}(z))
$$

with $\tilde{w}(0)=0$, and vice versa.

Proof. a) Let $\varphi$ be as in the assumption, then the set of all local analytic solutions $f$ of (1) with $f(0)=0$ can, according to Theorem 3 , be represented in the form $f(x)=\tilde{f}_{0}\left(c_{k} x^{k}\right),|x|<\varepsilon(f)$, if $f(x)=c_{k} x^{k}+\cdots$, 
where $\tilde{f}_{0}$ is uniquely determined by $\varphi$ and $\tilde{f}_{0}(y)=y+\cdots$. This yields, by the chain rule,

$$
x f^{\prime}(x)=\tilde{f}_{0}\left(c_{k} x^{k}\right) k c_{k} x^{k}
$$

in some neighbourhood of 0 . Since $\tilde{f}_{0}^{-1}$ exists, we get

$$
x f^{\prime}(x)=k \tilde{f}_{0}^{\prime}\left(\tilde{f}_{0}^{-1}(f(x))\right) \tilde{f}_{0}^{-1}(f(x)),
$$

and introducing $N_{\varphi}:=\left(\tilde{f}_{0}^{\prime} \circ \tilde{f}_{0}^{-1}\right) \cdot \tilde{f}_{0}^{-1}$ we obtain

$$
x f^{\prime}(x)=k N_{\varphi}(f(x)) \text {, }
$$

i.e., Eq. (36), in some neighbourhood of 0 , where $N_{\varphi}(y)+\cdots$ is local analytic in $y=0$, since $\tilde{f}_{0}, \tilde{f}_{0}^{-1}$ and $\tilde{f}_{0}^{-1}$ are so.

Now we have to prove that each local analytic solution $w$ with $w(0)=0$ of (36) is a solution of (1). An easy calculation shows that each such solution $w$ of (36) is of the form $w(x)=c_{k} x^{k}+\cdots$ with some $c_{k} \in \mathbb{C}$, and that $w$ is uniquely determined by $c_{k}$ (we omit the details). On the other hand, by the construction of (36) there exists a solution $w$ of (36) of the form $w(x)=c_{k} x^{k}+\cdots$, namely the solution $x \mapsto \tilde{f}_{0}\left(c_{k} x^{k}\right)$ of (1) from which we started.

It remains to show that there is only one $N$, such that (1) and

$$
x w^{\prime}(x)=k N(w(x)), \quad w(0)=0
$$

have the same set of local analytic solutions.

Assume that

$$
x^{\prime} w(x)=k N_{j}(w(x)), \quad j=1,2, \quad w(0)=0,
$$

with local analytic functions $N_{j}$, such that $N_{j}(x)=x+\cdots$ have the same set of local analytic solutions as (1). Then there is a local analytic function $w(x)=c_{k} x^{k}+\cdots, k \geq 1, \quad c_{k} \neq 0$ which satisfies both differential equations and hence we get by substitution, $k\left(N_{1}-N_{2}\right)(w(x))=0$ in some neighbourhood of 0 . Since the values $w(x)$ cover a neighbourhood of 0 , since $w \neq 0$, we find by the identity theorem $N_{1}-N_{2}=0$.

b) The proof is similar as for a). This completes the proof of Theorem 5.

There again an inverse problem arises: Given $N, N(x)=x+\cdots$, analytic in $x=0$. Does there exist a $\varphi, \varphi(x)=x^{k+1}+\cdots$, such that the set of local analytic solutions $w$ of (41) is the same as the set of local analytic solutions $f$ of (1)? The answer is positive and given by 
Theorem 6. Let $N(x)=x+\cdots$ be analytic in $x=0$. Then there exists a unique $\varphi$, local analytic at $x=0, \varphi(x)=x^{k+1}+\cdots$ such that the Briot-Bouquet differential equation

$$
x w^{\prime}(x)=k \cdot N(w(x)), \quad w(0)=0
$$

has the same set of local analytic solutions as (1). The function $N_{\varphi}$, associated with $\varphi$ according to Theorem 5, equals $N$.

Proof. From the theory of Briot-Bouquet differential equations (see in particular [8], pp. 160-162) we deduce that all formal solutions $w(x)$ with $w(0)$ of $(43)$ are given by $w(x)=c_{k} x^{k}+\cdots$, where $c_{k} \in \mathbb{C}$ can be arbitrarily prescribed, and that the formal solution $w(x)=c_{k} x^{k}+\cdots$ is uniquely determined by $c_{k}$. Moreover, each $w(x)$ is convergent in some neighbourhood of 0 , so that the formal solutions of (43) yield all local analytic $w$ solutions of (43) with $w(0)=0$. For the convenience of the reader we give another, direct approach to the local analytic solutions $w(x)$ with $w(0)=0$ of (36).

First of all, it follows from (36) by comparing coefficients on both sides that such a $w$ has the form $w(x)=c_{k} x^{k}+\cdots$, where $c_{k} \in \mathbb{C}$ is not determined whereas the coefficients of $c_{\nu}, \nu \geq k+1$, are uniquely determined by $c_{k}$ and they exist. If $c_{k}=0$ we obtain $w=0$. Now let $c_{k} \neq 0$. Then $w(x)=c_{k} x^{k} \tilde{w}(x)$ with $\tilde{w}(x)=1+\gamma_{1} x+\cdots$. Hence (43) is in a neighbourhood of 0 equivalent with

$$
c_{k} x^{k+1} \tilde{\boldsymbol{w}}^{\prime}(x)=\sum_{\nu \geq 2} k \delta_{\nu}\left(c_{k} x^{k}\right)^{\nu} \tilde{\boldsymbol{w}}(x)^{\nu},
$$

where we use $N(y)=y+\delta_{2} y^{2}+\cdots$. If $x \neq 0$ we deduce from that

$$
\tilde{w}^{\prime}(x)=\sum_{\nu \geq 2} k \delta_{\nu} c_{k}^{\nu-1} x^{(\nu-1) k-1} \tilde{w}(x)^{\nu}, \quad \tilde{w}(0)=1,
$$

which holds in a punctured neighbourhood of 0 . We show that it holds in a neighbourhood of $x=0$.

To see this we firstly prove that the function

$$
(x, w) \longmapsto \sum_{\nu \geq 2} k \delta_{\nu} c_{k}^{\nu-1} x^{(\nu-1) k-1} w^{\nu}
$$

is defined and holomorphic in some region $|x|<\varepsilon_{1},|w-1|<\varepsilon_{2}$. From the convergence of $N(y)=y+\sum_{\nu \geq 2} \delta_{\nu} y^{\nu}$ we get, by simple estimations, that the series in (46) is indeed absolutely convergent if $|x|<\rho_{1}$ and $|w-1|<\rho_{2}$, for some $\rho_{1}, \rho_{2}>0$. Hence it is uniformly convergent in each compact set contained in $|x|<\rho_{1},|w-1|<\rho_{2}$, and by 
Weierstrass' theorem it represents a function holomorphic in $|x|<\rho_{1}$, $|w-1|<\rho_{2}$. Hence we are allowed to substitute for $w$ the convergent series $\tilde{w}(x)=1+\tilde{\gamma}_{1} x+\cdots$ for sufficiently small $|x|$ into the series in (46), and obtain an analytic function in some neighbourhood of 0 . This means that the differential equation for $\tilde{w}$ can be continued to hold also in $x=0$, and that it allows the application of Cauchy's existence and uniqueness theorem. Hence, $\tilde{w}(x)$ is indeed analytic at $x=0$, and the existence result for (43) holds.

Then we consider the Briot-Bouquet differential equation

$$
x \tilde{W}^{\prime}(x)=N(\tilde{W}(x)), \quad \tilde{W}(0)=0 .
$$

Here we get from the general theory [7] that (47) has exactly one formal solution $\tilde{w}_{0}$ with $\tilde{w}_{0}(x)=x+\cdots$ which is also convergent in some neighbourhood of 0 . We will now prove that, using this $\tilde{w}_{0}$, we get the local analytic solutions of (47) in the form

$$
w(x)=\tilde{w}_{0}\left(c_{k} x^{k}\right),
$$

where $|x|$ is sufficiently small and $c_{k}$ can be arbitrarily chosen in $\mathbb{C}$. We deduce from (48) and (47) that

$$
x^{\prime} w(x)=x \tilde{w}_{0}^{\prime}\left(c_{k} x^{k}\right) \cdot k \cdot c_{k} x^{k-1}=k c_{k} x^{k} \tilde{w}_{0}^{\prime}\left(c_{k} x^{k}\right)=k N(w(x))
$$

i.e., Eq. (43) is satisfied in some neighbourhood of 0 , where $w(x)=c_{k} x^{k}+\cdots$. So $w(x)$ is the unique solution of (43) with $w(x)=c_{k} x^{k}+\cdots$. Since $c_{k} \in \mathbb{C}$ is arbitrary (48) yields all local analytic solutions of (43). By Theorem 4a) there exists a unique $\varphi(x)=$ $x^{k+1}+\cdots$ such that $\left\{w ; w(x)=\tilde{w}_{0}^{\prime}\left(c_{k} x^{k}\right), c_{k} \in \mathbb{C},|x|<\varepsilon(w)\right\}$ is the set of all local analytic solutions of (1). By Theorem $5 \mathrm{a}, N$ is the unique function such that the set of all local analytic solutions of (43) coincides with the set of such solutions of (1), so $N=N_{\varphi}$.

Remark 7. The proof of Theorems 1 and 3 on the existence of local analytic solutions of (1) and the structure of these solutions may be entirely built on the corresponding results for Briot-Bouquet differential equations. This has the advantage that the proof of convergence of formal solutions of a Briot-Bouquet differential equation by Cauchy's method of majorant, is rather short and elegant. So the existence results (Theorem 1) and the results on the structure of solutions (Theorem 3, Theorem 4) can be deduced for formal solutions of (1), neglecting the convergence, and the same holds for the connection of (1) with Briot-Bouquet differential equations. From the convergence of any formal solution of a Briot-Bouquet equation, provided 
the given series $N=N_{\varphi}$ is convergent, we obtain, going back, the convergence of all formal solutions of (1).

\section{Generalized Dhombres Equations and Iteration Groups}

There are also close relations of generalized Dhombres equations (1) to iteration groups. In order to present these relations we recall the basic definitions and properties of analytic iteration groups of local analytic functions and their description by Aczél-Jabotinsky differential equations (see [8], [9], [10], also [15]).

A family $\left(F_{t}\right)_{t \in \mathbb{C}}$ of formal power series $F_{t}(x)=c_{1}(t) x+\cdots$ with $c_{1}(t) \neq 0(t \in \mathbb{C})$ is an analytic iteration group, if all coefficient functions $t \mapsto c_{\nu}(t)$ are entire functions and if the translation equation

$$
F_{t+s}(x)=F_{t}\left(F_{s}(x)\right), \quad(t, s \in \mathbb{C})
$$

holds. In particular we will consider the case when each $F_{t}$ is a convergent power series. Then for each $\left(t, s \in \mathbb{C}^{2}\right)$ there exists a neighbourhood of $z=0$ such that

$$
F_{t+s}(z)=F_{t}\left(F_{s}(z)\right)
$$

holds in this neighbourhood. If $c_{1}(t)=e^{\lambda t}, t \in \mathbb{C}$, with $\lambda \neq 0$, then the family $\left(F_{t}\right)_{t \in \mathbb{C}}$ is an iteration group of type I. We have the following characterization of iteration groups of type I of formal series.

If $\left(F_{t}\right)_{t \in \mathbb{C}}$ is such an iteration group of type I, then there exists a formal series $H(x)=x+h_{2} x^{2}+\cdots$ such that the set of formal solutions $\Phi(x)=\gamma_{1} x+\cdots, \gamma_{1} \neq 0$, of the Aczél-Jabotinsky differential equation

$$
H(x) \frac{d \Phi}{d x}(x)=H(\Phi(x))
$$

is exactly the family $\left(F_{t}\right)_{t \in \mathbb{C}}$, considered as a set. Conversely, the set of all formal solutions $\Phi$ of (52), with $H(x)=x+h_{2} x^{2}+\cdots$ may be parameterized as $\left(F_{t}\right)_{t \in \mathbb{C}}$, where $\left(F_{t}\right)_{t \in \mathbb{C}}$ is an iteration group of type I, uniquely determined if we take $\lambda=1$.

Furthermore, all series $F_{t}$ of an iteration group $\left(F_{t}\right)_{t \in \mathbb{C}}$ are convergent if and only if the corresponding $H$ is convergent. This means that $H$ is a convergent series if and only if all formal solutions $\Phi$ of (52) are convergent. However, there need not exist a neighbourhood of 0 where all formal solutions of (52) are convergent.

Now we explain the connection of Aczél-Jabotinsky differential equations (54), where $H(x)=x+h_{2} x^{2}+\cdots$, with certain Briot- 
Bouquet differential equations, namely precisely those which were used in Sect. 4. Let $\Psi^{\prime}$ be the derivative of an analytic function $\Psi$.

Lemma 1. Let $H(x)=x+h_{2} x^{2}+\cdots$ be analytic at $x=0$. Let $B$ be the unique local analytic solution of

$$
x B^{\prime}(x)=H(B(x))
$$

with $B(x)=x+\cdots$. Then for each local analytic solution $\Phi$, $\Phi(x)=\gamma_{1} x+\cdots$, of

$$
H(x) \Phi^{\prime}(x)=H(\Phi(x))
$$

$\Psi:=\Phi \circ B$ is a solution of

$$
x \Psi^{\prime}(x)=H(\Psi(x)) .
$$

Conversely, if $\Psi(x)=\gamma_{1} x+\cdots$ is a solution of (55), then $\Phi:=$ $\Psi \circ B^{-1}$ is a solution of (54).

Proof. Let $B$ be defined as above. Then, for a solution $\Phi$ of (54), $\Psi:=\Phi \circ B$ satisfies the differential equation

$$
x B^{\prime}(x)=H(B(x)) .
$$

Indeed, putting $A=B^{-1}, \Phi=\Psi \circ A$, we get from (54), using the chain rule,

$$
H(x) \cdot\left(\Psi^{\prime}\right)(A(x)) \cdot A^{\prime}(x)=H(\Phi(A(x))),
$$

in a neighbourhood of 0 . From $A(B(x))=x$ we obtain $A^{\prime}(B(x))=$ $\left(B^{\prime}(x)\right)^{-1}$, so that we have

$$
\left(\left(B^{\prime}(x)\right)^{-1} \cdot H(B(x))\right) \cdot \Phi^{\prime}(x)=H(\Phi(x)) .
$$

Since $B$ is a solution of (56) we finally get (55). The converse is proved in the same way which completes proof of Lemma 1.

Now, let again $\phi(x)=x^{k+1}+\cdots$ be given. By Theorem 5a) we associate to $\varphi$ the local analytic function $N_{\varphi}, N_{\varphi}(x)=x+\cdots$ such that the set of all local analytic solutions of (1) (Theorem 3 ) is exactly the set of solutions of (37). We may hence apply Lemma 1 to $N_{\varphi}$ in place of $H$ and obtain the relation between equations (1) and iteration groups.

Theorem 7. a) Let $\varphi(x)=x^{k+1}+d_{k+2} x^{k+2}+\cdots$ be analytic at $x=0$. The set of all nonzero local analytic solutions $f, f(0)=0$, of (1) can be described as a family

$$
f^{*}(t, x):=F\left(t, B\left(x^{k}\right)\right), \quad(t \in \mathbb{C}),
$$


where $B(x)=x+\cdots$ is analytic at $x=0$ and $(F(t, \cdot))_{t \in \mathbb{C}}$ is an analytic iteration of type I, i.e.,

$$
F(t, x)=e^{t} x+\cdots, \quad(t \in \mathbb{C}) .
$$

b) Let $N_{\varphi}$ be the local analytic function associated to (1) by Theorem 5. Then the analytic iteration group representing the nonzero solutions of (1) according to a) is given as the set of nonzero solutions $\Phi, \Phi(x)=\gamma_{1} x+\cdots$ of the Aczél-Jabotinsky equation

$$
N_{\varphi}(x) \cdot \phi^{\prime}(x)=N_{\varphi}(\varphi(x))
$$

with $\phi(x)=F(t, x)$ for $\gamma_{1}=e^{t}, t \in \mathbb{C} . B$ is the unique solution of

$$
x \cdot B^{\prime}(x)=N_{\varphi}(B(x))
$$

with $B(x)=x+\cdots$.

c) There is only one analytic iteration group $(F(t, x))_{t \in \mathbb{C}}$ with $F(t, x)=e^{t} x+\cdots(t \in \mathbb{C})$ and only one $B$ such that the set of all nontrivial analytic solutions of (1) has a representation (48).

Proof. a) and b) Under our hypothesis there exists, according to Theorem 5, a unique $N_{\varphi}, N_{\varphi}(x)=x+\cdots$, analytic at $x=0$, such that the set of nonzero solutions $\tilde{f}$ of

$$
x \tilde{f}^{\prime}(x)=N_{\varphi}(\tilde{f}(x)), \quad \tilde{f}(0)=0,
$$

is the set of local analytic functions yielding the representation $f(x)=\tilde{f}\left(x^{k}\right)$ of nonzero solutions of (1). By Lemma 1, writing now $N_{\varphi}$ for $H, \tilde{f}$ for $\Psi$ and $f^{*}$ for $\Phi$, there exists $\tilde{f}_{0}, \tilde{f}_{0}(x)=x+\cdots$, such that $\tilde{f}_{0}$ is a solution of (63) and such that $f^{*}:=\tilde{f} \circ \tilde{f}_{0}^{-1}$ is a solution of

$$
N_{\varphi}(x) \cdot\left(f^{*}\right)^{\prime}(x)=N_{\varphi}\left(f^{*}(x)\right) .
$$

All local analytic solutions $f^{*}$ of (64) can be obtained in this form from a solution $\tilde{f}$ of (63). From the introducing explanations of Sect. 5 we know that (61) determines a unique analytic iteration group of type I of the form

$$
F(t, x)=e^{t} x+\cdots \quad(t \in \mathbb{C}),
$$

where the local analytic functions $F(t, x)$ yield all nonzero solutions of (61). Going back we obtain the representation

$$
f^{*}(t, x):=F\left(t, B\left(x^{k}\right)\right), \quad(t \in \mathbb{C}),
$$

for the set of nontrivial local analytic solutions of (1). Hence a) and b) are proved. 
c) This follows from uniqueness results for Aczél-Jabotinsky and Briot-Bouquet differential equations. We omit the details. This completes the proof of Theorem 7.

Remark 8. From Theorem 7 we deduce that the set of nontrivial local analytic solutions of (1), when parametrized as in Theorem 7a) form a perturbation of an analytic iteration group. We find, in the case $k=1$,

$$
f^{*}(t+s, x)=f^{*}\left(t, B^{-1}\left(f^{*}(s, x)\right)\right), \quad(t, s \in \mathbb{C})
$$

in some neighbourhood of $x=0$, depending on $t$ and $s$.

Indeed, by (48) and since $(F(t, x))_{t \in \mathbb{C}}$ is a solution of the translation equation we get

$$
\begin{aligned}
f^{*}(t+s, x) & =F(t+s, B(x))=F(t, F(s, B(x))) \\
& =F\left(t, f^{*}(s, x)\right)=f^{*}\left(t, B^{-1}\left(f^{*}(s, x)\right)\right)
\end{aligned}
$$

in some neighbourhood of $x=0$.

If $k \geq 1$ we get, in a similar way,

$$
f^{*}(t+s, x)=f^{*}\left(t,\left(B^{-1}\left(f^{*}(s, x)\right)^{1 / k}\right), \quad(t, s \in \mathbb{C}),\right.
$$

where any of the $k$-th roots of $B^{-1}\left(f^{*}(s, x)\right)$, analytic at $x=0$, may be taken on the right-hand side.

Here we have again a converse problem: Let $(F(t, x))_{t \in \mathbb{C}}$ be an analytic iteration group of type $\mathrm{I}, F(t, x)=e^{t} x+\cdots(t \in \mathbb{C})$, whose elements are local analytic at $x=0$, and let $k \geq 1$. Does there exist $\varphi(x)=x^{k+1}+\cdots$, such that the nontrivial solutions of (1) can be represented by (66), using this iteration group and an appropriate $B$ ? The answer is affirmative and given by

Theorem 8. Let $k \geq 1$ and let $(F(t, x))_{t \in \mathbb{C}}$ be an analytic iteration group of local analytic functions $F(t, \cdot), F(t, x)=e^{t} x+\cdots(t \in \mathbb{C})$. Then there exists a unique $\varphi, \varphi(x)=x^{k+1}+d_{k+2} x^{k+2}+\cdots$, analytic at $x=0$, and a unique $B(x)=x+\cdots$, such that the nonzero solutions of (1) can be presented in the form (66).

Proof. By what has been said at the beginning of Sect. 5, there exists for $(F(t, x))_{t \in \mathbb{C}}$ a unique $H(x)=x+h_{2} x^{2}+\cdots$, analytic at $x=0$, such that (54) has exactly the nontrivial local analytic solutions $\Phi$ of the form $x \mapsto F(t, x)$. By Lemma 1 there exists a (unique) $B$, $B(x)=x+\cdots$ such that via $\Psi:=\Phi \circ B^{-1}$, (54) is equivalent with (55); note that $B$ is the unique solution of (55) with $B(x)=x+\cdots$. Then, according to Theorem 6 and its proof there exists a unique local analytic function $\varphi, \varphi(x)=x^{k+1}+\cdots$, such that the set of all local 
analytic solutions $\tilde{f}:=\Psi$ of (55) yields the set of all local analytic solutions of (1) in the form

$$
f(x)=\tilde{f}\left(x^{k}\right), \quad|x|<\varepsilon(f) .
$$

Moreover, using the notation of Theorem 5 we have $H=N_{\varphi}$. Then, going back to $\Phi$, we find altogether for $f$ the representation (66).

The assertions refering to uniqueness follow from the arguments used before. This completes the proof of Theorem 8 .

In Remark 8 we found for the nonzero solutions of (1) in the case $\varphi(x)=x^{2}+\cdots$ the composition law (68), in some neighbourhood of 0 , depending on $s$ and $t$. We may consider this as a perturbed translation equation. However, in this perturbation of the translation equation, $B$ was not arbitrary, but was uniquely determined by $\varphi$. Now let $C, C(x)=x+c_{2} x^{2}+\cdots$, be an arbitrary local analytic function and $\left(f^{*}(t, x)\right)_{t \in \mathbb{C}}$ be a family of invertible local analytic functions satisfying

$$
f^{*}(t+s, x)=f^{*}\left(t, C^{-1}\left(f^{*}(s, x)\right)\right), \quad(t, s \in \mathbb{C}),
$$

in some neighbourhood of 0 , depending on $s$ and $t$. Then

$$
F(t, x):=f^{*}\left(t, C^{-1}(x)\right), \quad(t \in \mathbb{C}),
$$

is an iteration group. It is of the form $F(t, x)=e^{t} x+\cdots(t \in \mathbb{C})$, if and only if $f^{*}(t, x)=e^{t} x+\cdots(t \in \mathbb{C})$. We assume now that $f^{*}(t, x)=$ $e^{t} x+\cdots$ and that all coefficient functions of $\left(f^{*}(t, x)\right)_{t \in \mathbb{C}}$ are entire. This implies that $(F(t, x))_{t \in \mathbb{C}}$ is an analytic iteration group of type I. We associate with this iteration group, according to Theorem 8 , the unique $\varphi, \varphi(x)=x^{2}+\cdots$ (since here $k=1$ ) such that all nonzero solutions of (1) are given by

$$
\bar{f}(t, x)=F(t, B(x)), \quad(t \in \mathbb{C}),
$$

with an appropriate and unique $B$. From (1) for $\bar{f}(t, x):=g(x)$ we obtain the functional equation

$$
g\left(D^{-1}(D(x) \cdot g(x))\right)=\varphi(g(x))
$$

with $D=B^{-1} \circ C$. This is another generalization of the Dhombres equation which coincides with (1) iff $B=C$.

These arguments may easily be extended to obtain

Remark 9. Let $C, C(x)=x+\cdots$, be analytic at $x=0$, and let $\left(f^{*}(t, x)\right)_{t \in \mathbb{C}}$ with $f^{*}(t, x)=e^{t} x+\cdots$ and entire coefficient functions be a solution of (71) in a neighbourhood of $x=0$, depending on $t$ and $s$ (i.e., a solution of a perturbated translation equation). 
Then there exists a unique $\varphi, \varphi(x)=x^{2}+\cdots$, and a unique $D$, $D(x)=x+\cdots$, such that the set of nonzero local analytic solutions $g$ with $g(0)=0$ of

$$
g\left(D^{-1}(D(x) \cdot g(x))\right)=\varphi(g(x))
$$

is given by the functions $x \mapsto f^{*}(t, x)$, for all $t$.

\section{Relations Between Two Generalized Dhombres Equations: An Application of the Böttcher Functional Equation}

The aim of this section is to give an explicit bijection of the set of local analytic solutions of a generalized Dhombres equation (1) with $\varphi:=\varphi_{1}$ to the set of local analytic solutions of another (1) with $\varphi:=\varphi_{2}$, if $\varphi_{j}(x)=x^{k+1}+\cdots, j=1,2$ with the same $k \geq 1$. This can be done by using the Böttcher functions $B_{\varphi_{j}}$ of $\varphi_{j}$, for $j=1,2$, and will also give another way to solve (1) in the local analytic situation.

It is well known (see e.g. [16], pp. 60-61) that to $\varphi(x)=x^{k+1}+\cdots$, analytic at $x=0$, there exists a unique local analytic and invertible $B_{\varphi}, B_{\varphi}(x)=x+\cdots$, such that

$$
B_{\varphi}^{-1}\left(B_{\varphi}(x)^{k+1}\right)=\varphi(x)
$$

in some neighbourhood of 0 . We call $B_{\varphi}$ Böttcher function of $\varphi$.

Using $B_{\varphi}$ we get another representation of the nontrivial local analytic solutions of (1), namely,

Theorem 9. Let $\varphi(x)=x^{k+1}+\cdots(k \geq 1)$ be analytic at $x=0$. Then the set of all nontrivial local analytic solutions $f$ of (1) with $f(0)=0$ is given by

$$
f(x)=B_{\varphi}^{-1}\left(\left(\left[\eta x \prod_{y=0}^{\infty} \frac{z^{k(k+1)^{\nu}}}{B_{\varphi}^{-1}\left(z^{k(k+1)^{\nu}}\right)}\right]^{[-1]}\right)^{k}\right)
$$

for $\eta \in \mathbb{C} \backslash\{0\},|x|<\varepsilon(\eta)$. Here $B_{\varphi}$ is the Böttcher function of $\varphi$ and $[\theta(x)]^{[-1]}$ denotes the inverse function of $\theta$.

The proof of Theorem 9 will be given together with that of Theorem 10 which establishes an explicit $1: 1$ correspondence between the sets of nontrivial local analytic solutions of two generalized Dhombres equations (1) for $\varphi:=\varphi_{1}$ and $\varphi:=\varphi_{2}$, if

$$
\varphi_{j}(x)=x^{k+1}+\cdots, \quad(j=1,2),
$$

with the same $k \geq 1$. 
Theorem 10. Let $\varphi_{j}, \varphi_{j}(x)=x^{k+1}+\cdots(j=1,2)$ be analytic at $x=0, k \geq 1$. Let $B_{\varphi_{j}}$ be the Böttcher function of $\varphi_{j}$, and denote by $f_{1}$ (resp. $f_{2}$ ) the local analytic solutions of (1) with $\varphi:=\varphi_{1}$ and $\varphi:=\varphi_{2}$, respectively, with the same initial part $\eta x(\eta \neq 0)$. Then we have

$$
f_{2}(x)=B_{\varphi_{2}}^{-1}\left(\left(\left(\prod_{\nu=0}^{\infty} \frac{B_{\varphi_{1}}^{-1}}{B_{\varphi_{2}}^{-1}}\left(z^{k(k+1)^{\nu}}\right)\right) \cdot\left[\left(B_{\varphi_{1}} \circ f_{1}\right)(x)^{1 / k}\right]^{[-1]}\right)^{[-1]}\right)^{k}
$$

in some neighbourhood of 0.

Proof of Theorems 9 and 10. Let (1) with $\varphi(x)=x^{k+1}+\cdots$ be given, and let $f$ be a solution of (1). Define $g:=B_{\varphi} \circ f$. Then using (76) and (1) we see that (1) is equivalent with

$$
g\left(x B_{\varphi}^{-1}(g(x))\right)=g(x)^{k+1}
$$

in some neighbourhood of 0 . We know already from the proof of Theorem 1 and from Theorem 2 that, to solve (80), it is sufficient to put $g(x)=T(x)^{k}$ or $g(x)=T\left(x^{k}\right)$ since this leads to solve (1) and hence also (80) because of $g=B_{\varphi} \circ f$. Let us follow the first possibility $g(x)=T(x)^{k}$. By well-known computations and arguments we obtain for $\mathcal{U}=T^{-1}$ the functional equation

$$
B_{\varphi}^{-1}\left(x^{k}\right) \cdot \mathcal{U}(x)=\mathcal{U}\left(x^{k+1}\right)
$$

with $\mathcal{U}(x)=u_{1} x+\cdots$, equivalent with (1). The advantage of (81) lies in the fact that the dependence on $\varphi$ is now concentrated in the first (known) factor on the left-hand side of (81), which holds in a neighbourhood of 0 . To (81) we may, as for the proof of Theorem 1, apply the existence and uniqueness results for generalized Böttcher equations (see [11]) which yields that to each $u_{1} \in \mathbb{C}$ there exists a unique solution $\mathcal{U}$ with $\mathcal{U}(x)=u_{1} x+\cdots$. Now we consider two generalized Dhombres equations (1), with $\varphi:=\varphi_{j}, j=1,2$, with $\varphi_{j}(x)=x^{k+1}+\cdots$, the corresponding Böttcher functions $B_{\varphi_{1}}$ and $B_{\varphi_{2}}$ and the associated equations (81).

Let us denote by $\mathcal{U}_{j}(\eta ; x)$ the unique local analytic solution of

$$
B_{\varphi_{j}}^{-1}\left(x^{k}\right) \cdot \mathcal{U}_{j}(x)=\mathcal{U}_{j}\left(x^{k+1}\right),
$$

with $\mathcal{U}_{j}(\eta ; x)=\eta x+\cdots, \eta \neq 0$. Let $E_{\eta}$ be the quotient $\mathcal{U}_{1} / \mathcal{U}_{2}$ which is, by holomorphic extension to $x=0$, analytic in some neighbourhood of 0 , also $E_{\eta}(x)=1+\cdots$. Similarly, we see the quotient 
$B_{\varphi_{2}}^{-1} / B_{\varphi_{1}}^{-1}$ as an analytic function around 0 , and have

$$
\frac{B_{\varphi_{2}}^{-1}}{B_{\varphi_{1}}^{-1}}(x)=1+\cdots .
$$

Dividing (82), side by side, we obtain

$$
\frac{B_{\varphi_{2}}^{-1}}{B_{\varphi_{1}}^{-1}}\left(x^{k}\right) \cdot E_{\eta}(x)=E_{\eta}\left(x^{k+1}\right)
$$

in some neighbourhood of 0 . Now, $E_{\eta}$ does not depend on $\eta$, since (83) has only one solution with absolute term 1 . So write $E_{\eta}=E$, where $E$ is uniquely determined by the pair $\left(\varphi_{1}, \varphi_{2}\right)$.

$E$ can be calculated from (83) by the classical "iteration process" for linear functional equations which gives

$$
E(x)=\prod_{\nu=0}^{\infty} \frac{B_{\varphi_{1}}^{-1}}{B_{\varphi_{2}}^{-1}}\left(x^{k(k+1)^{\nu}}\right)
$$

converging uniformly and absolutely in each compact subset of a certain neighbourhood of $x=0$. Using the definition $E=\mathcal{U}_{2} / \mathcal{U}_{1}$ we get from (84)

$$
\mathcal{U}_{2}(\eta, x)=\left(\prod_{\nu=0}^{\infty} \frac{B_{\varphi_{1}}^{-1}}{B_{\varphi_{2}}^{-1}}\left(x^{k(k+1)^{\nu}}\right)\right) \mathcal{U}_{1}(\eta, x) .
$$

If we take here $\varphi_{1}(x)=x^{k+1}, \varphi_{2}(x)=: \varphi(x)$, then $B_{\varphi_{1}}(x)=x$, $B_{\varphi_{2}}=: B_{\varphi}, \mathcal{U}_{1}(\eta, x)=\eta x$. Hence (85), together with $T=\mathcal{U}^{-1}$, $g=T^{k}, B_{\varphi}^{-1} \circ g=f$ leads to Theorem 9. In a similar way we obtain Theorem 10.

\section{Acknowledgement}

The research was supported, in part, by projects 201/03/1153 from the Czech Science Foundation, MSM4781305904 from the Czech Ministry of Education, and by the program AKTION Österreich-Tschechische Republik. Support of these institutions is gratefully acknowledged.

\section{References}

[1] Dhombres, J. (1975) Applications associatives ou commutatives. C. R. Acad. Sci. Paris Sér. A 281: 809-812

[2] HILle, E. (1976) Ordinary Differential Equations in the Complex Domain. John Wiley, New York

[3] INCE, E. L. (1956) Ordinary Differential Equations (Reprint 1956). Dover Publications, New York 
[4] Kahlig, P., Smítal, J. (2001) On a generalized Dhombres functional equation. Aequationes Math. 62: 18-29

[5] KAHLIG, P., SMÍTAL, J. (2002) On a generalized Dhombres functional equation, II. Mathem. Bohem. 127: 547-555

[6] Kuczma, M., Choczewski, B., Ger, R. (1994) Iterative Functional Equations (Encyclopedia of Mathematics and Its Applications, Vol. 32). Cambridge University Press, Cambridge

[7] LAINE, I. (2003) Introduction to local theory of complex differential equations. In: LAINE, I. (ed.) Complex Differential and Functional Equations (Mekrijärvi, 2000), pp. 81-106. University of Joensuu, Department of Mathematics, Report Series No. 5, Joensuu

[8] REICH, L. (1986) Holomorphe Lösungen der Differentialgleichung von E. Jabotinsky. Sitzungsber. Österr. Akad. Wiss. Wien, Math.-nat. Kl. Abt. II 195: $157-166$

[9] REICH, L. (1986) On a differential equation arising in iteration theory of rings of formal power series in one variable. In: LIEDL, R., et al. (eds.) Iteration Theory and Its Functional Equations (Lecture Notes in Math., Vol. 1163), pp. 135-148. Springer, Berlin Heidelberg New York

[10] REICH, L. (1988) Die Differentialgleichungen von Aczél-Jabotinsky, von BriotBouquet und maximale Familien konvergenter vertauschbarer Potenzreihen. In: Withalm, C. (ed.) Complex Methods in Partial Differential Equations, pp. 137-150. Akademie-Verlag, Berlin

[11] REICH, L. (2004) Generalized Böttcher equations in the complex domain. In: Aulaskari, R., et al. (eds.) Symposium on Complex Differential and Functional Equations, Joensuu, Department of Mathematics, Report Series No. 6, pp. 135-147

[12] Reich, L., SMítal, J., ŠTEFÁnKovÁ, M. (2004) The continuous solutions of a generalized Dhombres functional equation. Mathem. Bohem. 129: 399-410

[13] Reich, L., Smítal, J., ŠtefánKovÁ, M. (2005) The converse problem of the generalized Dhombres functional equation. Mathem. Bohem. 130: 301-308

[14] REMmerT, R. (1992) Funktionentheorie 1, 3. Aufl. Springer, Berlin Heidelberg New York

[15] Scheinberg, St. (1970) Formal power series in one variable. J. Math. Anal. Appl. 31: 321-333

[16] Steinmetz, N. (1993) Rational Iteration. Complex Analytic Dynamical Systems (de Gruyter Studies in Math., Vol. 16). Walter de Gruyter, Berlin

Authors' addresses: Prof. Ludwig Reich, Institut für Mathematik, Karl-FranzensUniversität Graz, 8010 Graz, Austria. E-Mail: ludwig.reich@uni-graz.at; Prof. Jaroslav Smítal, Prof. Marta Štefánková, Mathematical Institute, Silesian University, 74601 Opava, Czech Republic. E-Mail: jaroslav.smital@math.slu.cz, marta.stefankova@ math.slu.cz. 